\title{
Optimal management of acute nonrenal adverse reactions to iodine-based contrast media
}

This article was published in the following Dove Press journal:

Reports in Medical Imaging

27 June 2013

Number of times this article has been viewed

\section{Yousef W Nielsen Henrik S Thomsen \\ Department of Radiology, Copenhagen University Hospital Herlev, Copenhagen, Denmark}

Correspondence: Yousef W Nielsen Department of Radiology 54E2, Copenhagen University Hospital Herlev, DK-2730 Herlev, Denmark Tel +45 51237991

Email ywnielsen@gmail.com
Abstract: Acute adverse reactions to iodine-based contrast media occur within 60 minutes of administration. The reactions range from mild (flushing, arm pain, nausea/vomiting, headache) to moderate (bronchospasm, hypotension), and severe (cardiovascular collapse, laryngeal edema, convulsions, arrhythmias). Most acute adverse reactions occur in an unpredictable manner. Use of the older group of ionic iodine-based contrast agents increases the risk of acute adverse reactions. Other risk factors include previous reactions to contrast media, asthma, and allergic conditions. The exact pathophysiology of the acute adverse reactions is unknown, but some of the reactions are pseudoallergic mimicking type 1 allergic reactions. As antibodies against contrast media have not been consistently demonstrated, the reactions are, in most cases, not truly allergic in nature. Most of the severe and fatal adverse reactions occur within the first 20 minutes after injection. Thus, it is important that patients are observed in the radiology department during this period. The radiologist should be near the room where contrast media is administered, and be ready to treat any acute nonrenal adverse reaction. Appropriate drugs and resuscitation equipment should be in/near the room where the contrast media is administered. The important first-line management of acute adverse reactions includes the establishment of an adequate airway, oxygen supplementation by mask, intravenous fluid administration, and measurement of blood pressure and heart rate. When severe anaphylactoid reactions occur, adrenaline should be given intramuscularly. Only one concentration of adrenaline $(1: 1000-1 \mathrm{mg} / \mathrm{mL})$ should be available in the radiology department to avoid dosing errors in stressful acute settings. Resuscitation team specialists should be the only ones giving intravenous adrenaline. It is important that all radiologists maintain the capability of performing first-line treatment of acute adverse reactions to contrast media.

Keywords: contrast media, adverse nonrenal reactions, treatment

\section{Introduction}

Although rare, severe acute adverse reactions to modern iodine-based contrast media are feared as they may have fatal outcomes; therefore, all radiologists should be prepared to give immediate treatment when acute contrast medium reactions occur. However, it has previously been shown that the acute treatment of severe reactions (anaphylactic/anaphylactoid) in radiology departments was deficient for both consultants and trainees. ${ }^{1}$ To avoid such insufficient management of acute adverse reactions, simple first-line treatment should be maintained by the radiologist and technicians/radiographers. The resuscitation team should handle the secondary management of acute adverse reactions, including the administration of supportive drugs. 
This review article gives an overview of acute nonrenal adverse reactions to iodine-based contrast media with an emphasis on treatment. The discussion of the incidence and pathophysiology of these adverse reactions refers to iodinebased contrast media used for computed tomography imaging and conventional radiography. However, treatment of acute adverse reactions is identical whether iodine-based or gadolinium-based (magnetic resonance imaging [MRI]) contrast agents cause them. Acute renal adverse reactions (contrast-induced nephropathy) are beyond the scope of this paper.

\section{Definition and classification}

An acute adverse reaction is defined as an adverse event occurring within 60 minutes of administration of a drug. The reactions are subdivided into mild, moderate, and severe. ${ }^{2}$ Mild reactions are indicated by flushing, arm pain, nausea/ vomiting, headache, pruritus, and mild urticaria. Some characteristics of this group of adverse reactions include that the reactions are usually of short duration and can be self-limiting. Specific treatment of mild reactions is rarely necessary. Some of these reactions are also seen in patients undergoing unenhanced imaging. Therefore, all mild reactions after enhanced imaging may not be due to the contrast agents, but due to the examination itself. Reactions due to the examination should not be registered as an acute adverse reaction to an agent. Moderate reactions include moderate cases of hypotension and bronchospasm. Furthermore, more serious degrees of the above-listed symptoms are classified as moderate reactions; these symptoms generally require appropriate treatment.

Severe reactions are life-threatening and comprise cardiovascular/pulmonary collapse, laryngeal edema, convulsions, cardiac arrhythmias, and unconsciousness. Aggravated manifestations of the symptoms listed under mild and moderate reactions are included in the group of severe reactions. Severe reactions prompt immediate highintensity treatment.

\section{Risk factors}

Although most acute adverse reactions to iodine-based contrast media occur in an unpredictable manner, some risk factors have been described in the literature. Patients with previous moderate or severe adverse reactions to iodine-based contrast agents are at increased risk. A large-scale study has shown a sixfold increase in adverse reactions for patients with previous severe reactions. ${ }^{3}$ The increased risk was present for both the older generation of ionic contrast agents and the more recent nonionic contrast agents. Another important risk factor is asthma, with a reported six- to tenfold increase in severe reactions. ${ }^{3}$ Interleukin-2 treatment (typically used in patients with malignant melanoma and renal cell carcinoma) also increases the risk of acute (and late) adverse reactions. Furthermore, allergic conditions that require medical treatment have been associated with an increased risk of acute adverse reactions. ${ }^{4}$ These observations have recently been confirmed in a large study of over 36,000 patients undergoing contrast-enhanced computed tomography with different nonionic agents. ${ }^{5}$ The study showed that six factors are predictors of acute adverse reactions: (1) a history of adverse reaction to contrast media; (2) a history of urticaria; (3) any drug allergy; (4) a contrast media concentration $>70 \%$; (5) patient age under 50 years; and (6) a total contrast media dose above $65 \mathrm{~g}$.

It is unclear whether or not administration of betaadrenergic blockers increases the risk of acute adverse reactions to contrast media. The literature has reported both increased and decreased risk in patients on beta-adrenergic blockers. ${ }^{6-8}$ Today, the administration of beta-adrenergic blockers is only rarely stopped before the injection of iodinebased contrast agents. ${ }^{9}$ The class of the iodine-based contrast agent is also important; the new generation of nonionic agents has a four- to fivefold lower risk of acute adverse reactions when compared with the older generation ionic agents. ${ }^{3}$

\section{Incidence}

Mild acute adverse reactions occur in up to $15 \%$ of patients after intravenous (IV) administration of ionic (high-osmolarity) iodine-based contrast media. Likewise, up

Table I Acute adverse reactions to contrast agents

\begin{tabular}{lll}
\hline Group & Reaction & $\begin{array}{l}\text { Intervention/ } \\
\text { treatment }\end{array}$ \\
\hline Mild & $\begin{array}{l}\text { Flushing } \\
\text { Arm pain }\end{array}$ & None \\
& Nausea/vomiting & \\
& Headache & \\
& Pruritus & \\
& Mild urticaria & Low-intensity \\
Moderate & Hypotension & treatment \\
& Bronchospasm & \\
& Serious degrees of the & High-intensity \\
& above-listed mild reactions & life-saving \\
Severe & Cardiovascular collapse & treatment \\
& Cardiac arrhythmias & \\
& Convulsions & \\
& Laryngeal edema & \\
& Pulmonary edema & \\
& Unconsciousness & \\
& Very serious degrees of the above-listed \\
& mild and moderate reactions &
\end{tabular}


to $3 \%$ of patients have mild reactions following injection of nonionic (low-osmolarity and iso-osmolarity) iodine-based contrast media. The incidence of reported adverse events depends on whether or not the patients are asked if they experienced anything during/after the contrast administration. The incidence is higher when questions are asked. Moderate and severe acute adverse reactions are much less common, with a reported incidence of $0.22 \%$ and $0.04 \%$, respectively, for ionic contrast media, and $0.04 \%$ and $0.004 \%$ for nonionic contrast media. A recent study found an incidence rate of $0.0005 \%-0.0008 \%$ for severe reactions for nonionic contrast media. ${ }^{5}$ Fatal reactions to both groups of iodine-based contrast media are very rare $(1: 170,000)$, with no difference in mortality reported between the two groups. ${ }^{3,10,11}$

Physiological factors may also influence the incidence of acute adverse reactions. In 1980, Lalli stated, "the most important factors in the production of contrast media reactions are the patient's fear and apprehension." 12 It is therefore of importance that the staff in the radiology department try to reduce the patient's anxiety before injecting contrast media. Another similar aspect is that whenever a new drug (including contrast media) is introduced, there is over-reporting of adverse events - the so-called Weber effect. ${ }^{13}$

\section{Pathophysiology}

Most acute adverse reactions to intravascular contrast media are considered to be idiosyncratic or pseudoallergic reactions. ${ }^{2}$ Idiosyncratic reactions are genetically determined, qualitatively abnormal reactions to drugs that are related to metabolic or enzyme deficiencies. Pseudoallergic reactions are similar to allergic reactions, but they lack immunological specificity, ie, there is nonspecific complement activation and histamine release mimicking type 1 allergic reactions. ${ }^{14}$ The acute adverse reactions occur in an unpredictable, dose-independent manner; the exact pathophysiological mechanism is not well understood. However, activation and inhibition of several enzyme systems have been implicated. Release of biological mediators, such as histamine, prostaglandins, bra-

Table 2 First-line drugs and instruments that should be in the examination room

Oxygen (including a mask for administration)

Adrenaline I: 1000

Antihistamine $\mathrm{HI}$ - suitable for injection

Atropine

Beta-2 agonist metered dose inhaler

Intravenous fluids $-0.9 \%$ saline

Anticonvulsive drugs (diazepam)

Sphygmomanometer dykinin, serotonin, leukotrienes, adenosine, and endothelin may be involved. So far, there is no conclusive evidence to indicate that acute adverse reactions to iodine-based contrast media are allergic in nature, since antibodies against contrast media have not been consistently demonstrated. ${ }^{15-17}$ The few true reactions may be confirmed through a skin test, which can also provide information about whether the reaction is in response to a single agent or to the whole class of contrast agents.

Chemotoxic effects may also be involved in acute adverse reactions. These effects are dependent on dose and physiological characteristics of the contrast agents (such as osmolarity, viscosity, protein binding, and so on). High-osmolarity agents can cause a shift of fluid from the intracellular to the extracellular compartment; this leads to cell dehydration, and to an increase in cell viscosity precipitating cell dysfunction. ${ }^{15,16}$ There is no evidence that heating contrast agents to $37^{\circ} \mathrm{C}$ prevents acute adverse reactions. $^{18}$

Some researchers have recommended the use of medical prophylaxis prior to the administration of contrast agents in order to reduce the risk of acute adverse reactions. However, there is no evidence that prednisolone and histamines, for example, have any prophylactic effect on reactions induced by nonionic agents (although this was shown to be the case for the ionic agents). ${ }^{19}$ Furthermore, despite prophylactic medication, breakthrough reactions are seen. These reactions are not more severe than the original reaction, but they underline the importance of being prepared to treat an acute adverse reaction 24 hours, 7 days a week.

\section{Registration of acute adverse reactions (warning)}

It is very important that acute adverse reactions are properly noted in the patient record, so that correct information is available prior to the next examination, allowing the radiologists to take appropriate precautions. We recommend that all reactions, which require medical treatment, are reported, whereas mild reactions not requiring any treatment should not be registered because they may not always derive from the administration of the contrast medium. The only exception is when skin test results have shown that there was a true reaction to the contrast agent.

\section{Treatment}

Conducting appropriate assessment of and providing treatment for acute adverse reactions to contrast media 
are essential skills that all radiologists and radiographers/ technicians should have and maintain. Most patients have reactions while they are still in the radiology department. It has been shown that $94 \%-100 \%$ of severe and fatal adverse reactions occur within 20 minutes of intravascular contrast administration. ${ }^{20}$ Therefore, the radiologist should remain near the patient for at least the first critical minutes after contrast injection, and should remain in the immediate vicinity for the next $30-45$ minutes. In cases where there is an increased risk of an adverse reaction (most importantly, in patients with previous reactions) the venous access should be left in place after contrast injection, so that any needed IV drugs can be given without delay.

Important first-line management includes the establishment of an adequate airway passage, oxygen supplementation, IV fluid administration, and the measurement of both blood pressure and heart rate. In addition, essential first-line drugs and emergency equipment should be available in (or very near) the examination room where contrast media are given. These drugs include adrenaline, antihistamine H1 (suitable for injection), atropine, beta-2 agonist metered dose inhaler, anticonvulsive drugs (diazepam), IV fluids $(0.9 \%$ saline), and a sphygmomanometer.

\section{The acute adverse contrast reaction - general means of treatment Oxygen}

The administration of oxygen is a key element in the initial treatment of acute adverse reactions to intravascular contrast media. Oxygen should be administered by mask, and not by nasal "prongs" that are less effective; a high flow rate (6-10 L/minute) is recommended. Chronic obstructive pulmonary disease does not contraindicate oxygen administration in an acute setting. In addition, continuous oxygen supply should be available in all examination rooms where intravascular contrast media is given. As a note of caution, in the extremely high magnetic field of MRI scanner rooms, portable oxygen containers made of metal can become life-threatening projectiles. Therefore, built-in oxygen supplies should be available in all MRI scanner rooms.

\section{Intravascular fluid administration}

Intravascular fluid administration is another important element in the first-line treatment of acute contrast reactions. In patients with life-threatening hypotensive reactions to contrast administration, IV fluids have been reported to be the most effective treatment; ${ }^{21}$ thus, starting IV fluids should have high priority when hypotensive reactions occur.
An appropriate regime is the administration of $0.9 \%$ saline. Giving IV fluids should be initiated before administering drug treatments.

\section{Adrenaline}

In the acute setting, adrenaline (epinephrine) is an effective drug for treating many serious contrast reactions. The pharmacodynamic effects of adrenaline are subdivided into alpha- and beta-agonistic effects. Increase of blood pressure and reversal of peripheral vasoconstriction are alpha-agonistic effects. These effects are very important in relation to acute contrast reactions, as they counteract hypotension and angioedema. Beta-agonistic effects can increase cardiac output and rate (positive inotropic and chronotropic effects), can result in the reversal of bronchoconstriction, and may increase intracellular levels of cyclic adenosine monophosphate. ${ }^{22}$ The increase in cyclic adenosine monophosphate is believed to suppress the release of biological mediators from inflammatory cells, including mast cells.

For ease of treatment in a stressful acute setting, only one concentration $(1: 1000-1 \mathrm{mg} / \mathrm{mL})$ of adrenaline should be available in the radiology department. Ampoules of different concentrations can lead to unintended high or low doses of adrenaline being administered to patients. The 1:1000 preparation should be given intramuscularly only by radiologists treating acute adverse reactions. The advantages of intramuscular administration include the fact that there is no need for venous access, this type of administration is easy to learn, and there is a greater margin of safety for this modality than with IV administration. A potential risk of IV administration of adrenaline is the occurrence of a hypertensive crisis. The preferred site for intramuscular administration should be the anterolateral aspect of the middle third of the thigh. Since the subcutaneous fat tissue may be extensive, the needle used for injection should be long enough to reach the muscle. ${ }^{23}$ Standard doses of adrenaline are recommended: $0.5 \mathrm{mg}$ in adults, $0.3 \mathrm{mg}$ in children between 6 and 12 years old, and $0.15 \mathrm{mg}$ in children under 6 years of age. ${ }^{23}$ The intramuscular dose of adrenaline can be repeated after 5 minutes if there is no improvement of the patient's condition, and if the specialist resuscitation team has not yet arrived.

\section{Antihistamines}

Antihistamines play a limited role in treating acute adverse contrast reactions. ${ }^{2,3}$ They are primarily used for the treatment of skin reactions. 


\section{Corticosteroids}

IV administration of corticosteroids should not be used in the first-line treatment of contrast reactions. However, standard doses of corticosteroids may be used in the secondline treatment to reduce delayed recurrent symptoms. When treating severe adverse events, the resuscitation team should be involved in the decision whether corticosteroids should be used or not.

\section{Inhaled bronchodilators}

Inhaled beta-2 agonists (for example, terbutaline) are used to treat bronchospasm. As the drugs are delivered directly to the airways, large local doses are obtained. There is minimal systemic absorption, and therefore minimal cardiovascular effects. $^{2}$

\section{Atropine}

Atropine blocks vagal stimulation of the cardiac conductive system. Larger doses of atropine (0.6-1 mg) are indicated because lower doses can be insufficient for treating bradycardia associated with contrast-media-induced vagal reactions. $^{24}$

\section{The acute adverse contrast reaction - treatment of specific reactions}

The following section presents simple step-by-step guidelines for the first-line treatment of acute adverse contrast reactions. The guidelines are adapted from the current version of the contrast media guidelines from the European Society of Urogenital Radiology (ESUR). ${ }^{4}$

\section{Generalized anaphylactoid reaction}

The clinical presentation of anaphylaxis includes the following: (1) sudden onset and rapid progression of symptoms; (2) life-threatening airway, breathing, and/or circulation problems; and (3) skin and/or mucosal changes such as flushing, urticaria, or angioedema. Recent exposure to contrast media supports the diagnosis. The initial treatment of anaphylaxis includes:

1. Calling for the resuscitation team;

2. Maintaining an adequate airway; suction the airway as needed;

3. Elevate the patient's legs if hypotensive;

4. Oxygen administration by mask (6-10 L/minute);

5. Intramuscular adrenaline administration (1:1000); $0.5 \mathrm{mg}$ $(0.5 \mathrm{~mL})$ in adults. Repeat as needed;

6. IV fluid administration (normal saline); and
7. Administration of an antihistamine H1-blocker (for example, diphenhydramine $25-50 \mathrm{mg}$ IV).

During the anaphylactoid reactions, tryptase is released from the mast cells into the circulatory system. Thus, measurement of serum tryptase levels can be used to establish the diagnosis in cases where anaphylaxis is suspected. At a minimum, one blood sample should be taken 1-2 hours after the reaction. In an ideal setting, three samples should be taken - during the reaction, 1-2 hours after the reaction, and 24 hours after the reaction.

\section{Hypotension}

Severe hypotension may be present without respiratory symptoms. Isolated hypotension is characterized by normal sinus rhythm and tachycardia. Vagal reactions also cause hypotension, but in these cases, electrocardiography will show bradycardia (heart rate $<60$ beats per minute) due to the excessive vagal stimulation of the heart, which is characteristic of these reactions. Hypotension is often defined as a systolic blood pressure $<80 \mathrm{mmHg}$. The exact mechanism of vagal reactions is unknown; however, anxiety seems to predispose patients to these reactions.

The initial treatment of hypotension includes the following steps:

1. Elevate the patient's legs;

2. Administer oxygen by mask (6-10 L/minute);

3. Administer IV fluids (normal saline) - up to $3 \mathrm{~L}$ may be required; finally,

4. In patients with vagal reactions (bradycardia): $0.6-1 \mathrm{mg}$ of atropine should be administered intravenously. If necessary repeat after 3-5 minutes, up to $3 \mathrm{mg}$ total in adults. In patients with isolated hypotension (tachycardia): intramuscular adrenaline (1:1000); $0.5 \mathrm{mg}$ $(0.5 \mathrm{~mL})$ should be administered in adults. Repeat as needed.

\section{Laryngeal edema}

It is important to note that laryngeal edema does not respond well to the inhalation of beta-2-agonists. Actually, these agents can worsen symptoms. Instead, treatment should include:

1. Oxygen administration by mask (6-10 L/minute); and

2. Intramuscular administration of adrenaline (1:1000); $0.5 \mathrm{mg}(0.5 \mathrm{~mL})$ in adults.

\section{Bronchospasm}

The treatment of bronchospasm may include:

1. Oxygen administration by mask (6-10 L/minute); 
2. Inhalation of a beta-2-agonist using a metered dose inhaler (two or three deep inhalations); and

3. Intramuscular adrenaline administration (1:1000); $0.3 \mathrm{mg}$ $(0.3 \mathrm{~mL})$ in adults with normal blood pressure. If the patient is hypotensive, then $0.5 \mathrm{mg}(0.5 \mathrm{~mL})$ can be administered.

\section{Nausea and vomiting}

These reactions are usually self-limited; however, they are important as they may be the initial signs of more severe acute adverse reactions. In a report of fatal reactions after IV urography performed with high-osmolar iodine contrast media, $15 \%-20 \%$ of fatal reactions began with nausea and vomiting. ${ }^{12}$ Therefore, all patients with these symptoms should be observed for any systemic reactions, and the IV access should be left in place. Antiemetic drugs may be used in severe protracted cases.

\section{Urticaria}

Treatment of these reactions is usually not necessary; however, as for nausea and vomiting, urticarial reactions may occur before more severe systemic reactions, and patients should be observed and the IV access should be left in place. In protracted cases, antihistamines (H1) can be given intramuscularly or intravenously. Intramuscular adrenaline $(0.3 \mathrm{mg})$ should be reserved for the most severe cases.

\section{Strategy to reduce the risk of acute adverse reactions}

Based on the established risk factors of acute adverse reactions to iodine-based contrast media, the ESUR has formulated a strategy to reduce the risk of acute adverse reactions. ${ }^{4}$ The key elements are presented below:

For all patients:

- Use nonionic contrast agents in all patients

- Keep patients in the radiology department for 30 minutes after contrast media injection

- Have drugs and equipment for resuscitation readily available.

For patients with an increased risk (previous reaction, asthma, allergy, administration of ionic contrast media):

- Consider using an alternative imaging test not requiring an iodine-based contrast medium (ie, unenhanced computed tomography, MRI with or without gadoliniumbased contrast media, or ultrasound)

- Use a different iodine-based contrast medium for previous reactors to contrast media
- Consider the use of premedication. Prednisolone (30 mg given orally) can be administered 12 and 2 hours before the contrast medium; however, it should be noted that the clinical evidence of the effectiveness of premedication is sparse. ${ }^{4}$

\section{Conclusion}

Acute adverse reactions to iodine-based contrast media range from mild reactions to fatal events. In order to reduce acute adverse reactions, all radiologists should be aware of the predisposing risk factors for these reactions. It is of utmost importance that all radiologists are capable of identifying acute adverse reactions and providing immediate first-line treatment before the arrival of the resuscitation team.

\section{Disclosure}

The authors report no conflicts of interest in this work.

\section{References}

1. Bartlett MJ, Bynevelt M. Acute contrast reaction management by radiologists: a local audit study. Australas Radiol. 2003;47(4): 363-367.

2. Thomsen HS. Management of acute adverse reactions. In: Thomsen HS, Webb JAW, editors. Contrast Media: Safety Issues and ESUR Guidelines. 2nd ed. Berlin; Springer-Verlag; 2009:53-59.

3. Katayama H, Yamaguchi K, Kozuka T, Takashima T, Seez P, Matsuura K. Adverse reactions to ionic and nonionic contrast media. A report from the Japanese Committee on the Safety of Contrast Media. Radiology. 1990;175(3):621-628.

4. European Society of Urogenital Radiology. ESUR guidelines on contrast media version 8.0. Vienna, Austria: European Society of Urogenital Radiology; 2013. Available from: http://www.esur.org/guidelines. Accessed March 13, 2013.

5. Kobayashi D, Takahashi O, Ueda T, Deshpande GA, Arioka H, Fukui T. Risk factors for adverse reactions from contrast agents for computed tomography. BMC Med Inform Decis Mak. 2013;13:18.

6. Lang DM, Alpern MB, Visintainer PF, Smith ST. Increased risk for anaphylactoid reaction from contrast media in patients on beta-adrenergic blockers with asthma. Ann Intern Med. 1991;115(4):270-276.

7. Lang DM, Alpern MB, Visintainer PF, Smith ST. Elevated risk of anaphylactoid reaction from radiographic contrast media is associated with both beta-blocker exposure and cardiovascular disorders. Arch Intern Med. 1993;153(17):2033-2040. Erratum in: Arch Intern Med. 1993;153(21):2412.

8. Greenberger PA, Meyers SN, Kramer BL, Kramer BL. Effects of betaadrenergic and calcium antagonists on the development of anaphylactoid reactions from radiographic contrast media during cardiac angiography. J Allergy Clin Immunol. 1987;80(5):698-702.

9. Morcos SK, Thomsen HS, Webb JAW; for Contrast Media Safety Committee of the European Society of Urogenital Radiology. Prevention of generalized reactions to contrast media: a consensus report and guidelines. Eur Radiol. 2001;11(9):1720-1728.

10. Thomsen HS, Dorph S. High-osmolar and low-osmolar contrast media. An update on frequency of adverse drugs reactions. Acta Radiol. 1993;34(3):205-209.

11. Thomsen HS, Bush WH Jr. Adverse effects of contrast media: incidence, prevention and management. Drug Saf. 1998;19(4):313-324.

12. Lalli AF. Contrast media reactions: data analysis and hypothesis. Radiology.1980;134(1):1-12. 
13. Weber JCP. Epidemiology of adverse reactions to nonsteroidal antiinflammatory drugs. In: Rainsford KD, Velo GP, editors. Side-Effects of Anti-Inflammatory Drugs, Advances in Inflammation Research. New York, NY: Raven Press: 1984:1-7.

14. Stacul F. Currently available iodinated contrast media. In: Thomsen HS, Muller RN, Mattrey RF, editors. Trends in Contrast Media. Berlin, Germany: Springer; 1999:71-72.

15. Almén T. The etiology of contrast medium reactions. Invest Radiol. 1994;29 Suppl 1:S37-S45.

16. Siegle RL. Mechanisms of reactions to contrast media. In: Dawson P, Cosgrove DO, Grainger RG, editors. Textbook of Contrast Media. Oxford, UK: Isis Medical Media Ltd; 1999:95-98.

17. Laroche D, Aimone-Gastin I, Dubois F, et al. Mechanisms of severe, immediate reactions to iodinated contrast material. Radiology. 1998;209(1):183-190.

18. Davenport MS, Wang CL, Bashir MR, Neville AM, Paulson EK. Rate of contrast material extravasations and allergic-like reactions: effect of extrinsic warming of low-osmolality iodinated CT contrast material to 37 degrees C. Radiology. 2012;262(2):475-484.

19. Dillman JR, Ellis JH, Cohan RH, Strouse PJ, Jan SC. Allergic-like breakthrough reactions to gadolinium contrast agents after corticosteroid and antihistamine premedication. AJR Am J Roentgenol. 2008;190(1): 187-190.
20. Shehadi WH. Death following intravascular administration of contras media. Acta Radiol Diagn (Stockh). 1985;26(4):457-461.

21. vanSonnenberg E, Neff CC, Pfister RC. Life-threatening hypotensive reactions to contrast administration: comparison of pharmacologic and fluid therapy. Radiology. 1987;162(1 Pt 1):15-19.

22. Hoffmann BB, Lefkowitz RJ. Catecholamines and sympathomimetic drugs. In: Gilman AG, Rall TW, Nies AS, Taylor P, editors. The Pharmacological Basis of Therapeutics. 8th ed. New York, NY: Pergamon; 1990:192-198.

23. Resuscitation Council (UK). Emergency treatment of anaphylactic reactions. London, UK: Resuscitation Council (UK); 2013. Available from: http://www.resus.org.uk/pages/reaction.htm. Accessed March 11, 2013.

24. Bush WH, McClennan BL, Swanson DP. Contrast media reactions: prediction, prevention and treatment. Postgrad Radiol. 1993;13(2):137-147.
Reports in Medical Imaging

\section{Publish your work in this journal}

Reports in Medical Imaging is an international, peer-reviewed, open access journal publishing original research, reports, reviews and commentaries on all areas of medical imaging. The manuscript management system is completely online and includes a very quick and fair peer-review system, which is all easy to use.

\section{Dovepress}

Visit http://www.dovepress.com/testimonials.php to read real quotes from published authors. 